\title{
Exact Periodic Wave, Bisoliton, and Various Breather Solutions for the Zakharov Equations
}

\author{
Heng Wang, ${ }^{1}$ Longwei Chen, ${ }^{1}$ Hongjiang Liu, ${ }^{2}$ and Shuhua Zheng ${ }^{1}$ \\ ${ }^{1}$ College of Statistics and Mathematics, Yunnan University of Finance and Economics, Kunming, Yunnan 650221, China \\ ${ }^{2}$ City and Environment College, Yunnan University of Finance and Economics, Kunming, Yunnan 650221, China \\ Correspondence should be addressed to Longwei Chen; 1187411801@qq.com
}

Received 17 July 2015; Revised 2 November 2015; Accepted 5 November 2015

Academic Editor: Masoud Hajarian

Copyright (c) 2015 Heng Wang et al. This is an open access article distributed under the Creative Commons Attribution License, which permits unrestricted use, distribution, and reproduction in any medium, provided the original work is properly cited.

The Zakharov equations, which involve the interactions between Langmuir and ion acoustic waves in plasma, are analytically studied. By using the method of Exp-function, the periodic wave, bisoliton, Akhmediev breather, Ma breather, and Peregrine breather of the Zakharov equations are obtained. These results presented in this paper enrich the diversity of solution structures of the Zakharov equations. Furthermore, based on the numerical simulations of these solutions, some physics analysis of bisolitons and various breathers are given.

\section{Introduction}

In this paper, we consider the following Zakharov equations:

$$
\begin{aligned}
& n_{t t}-c_{s}^{2} n_{x x}=\beta\left(|E|^{2}\right)_{x x}, \\
& i E_{t}+\alpha E_{x x}=\delta n E,
\end{aligned}
$$

where $n$ is the perturbed number density of the ion (in the low-frequency response), $E$ is the slow variation amplitude of the electric field intensity, $c_{s}$ is the thermal transportation velocity of the electron-ion, $\alpha \neq 0, \beta \neq 0$, and $\delta \neq 0$ [1]. The Zakharov equation is one of the classical models describing the interactions between high- and low-frequency waves and governing nonlinear dynamics of travelling waves. In the interaction of laser-plasma, (1) plays crucial roles. In recent years, many exact solutions of (1) have been successfully obtained by different methods in [2-7].

Recently, some important mathematical physics equations have been widely studied [8-12]. In particular, the investigation of the rogue wave phenomenon of nonlinear partial differential equation achieved some significant advances.
Many rogue waves have been obtained [13-18]. Here we continue to analyze the rogue wave phenomenon and study the existence of rogue waves of (1).

Firstly, we set $\xi=k_{1} x+k_{2} t ; k_{1}$ and $k_{2}$ are arbitrary real constants. Equation (1) is transformed into the following equations:

$$
\begin{aligned}
n & =\frac{\beta k_{1}^{2}}{k_{2}^{2}-c_{s}^{2} k_{1}^{2}}|E|^{2}, \\
i E_{t}+\alpha E_{x x} & =\delta n E .
\end{aligned}
$$

Next (2) is reduced to the $(1+1)$-dimensional nonlinear Schrödinger equation:

$$
i E_{t}+\alpha E_{x x}+\gamma|E|^{2} E=0
$$

where $\gamma=-\delta \beta k_{1}^{2} /\left(k_{2}^{2}-c_{s}^{2} k_{1}^{2}\right)$. 
Then, to find the exact solutions of the Zakharov equations, we assume that

$$
E(x, t)=r e^{\left(i r^{2} \gamma t\right)}\left(1+\frac{A(x, t)+i B(x, t)}{C(x, t)}\right)
$$

where $r$ is a real constant. Substituting (4) into (3), we have

$$
\begin{aligned}
& 2 \gamma r^{2} A(x, t) C(x, t)^{2}+2 \alpha A(x, t) C_{x}(x, t)^{2} \\
& +\gamma r^{2} A(x, t)^{3}-2 \alpha A_{x}(x, t) C_{x}(x, t) C(x, t) \\
& \quad+\alpha A_{x x}(x, t) C(x, t)^{2}-\alpha A(x, t) C_{x x}(x, t) C(x, t) \\
& +B(x, t) C(x, t) C_{x}(x, t)+3 \gamma r^{2} A(x, t)^{2} C(x, t) \\
& +\gamma r^{2} B(x, t)^{2} C(x, t)-B_{t}(x, t) C(x, t)^{2} \\
& +\gamma r^{2} A(x, t) B(x, t)^{2}+i\left(\gamma r A(x, t)^{2} B(x, t)\right. \\
& \quad-A(x, t) C(x, t) C_{x}(x, t) \\
& \quad-\alpha B(x, t) C_{x x}(x, t) C(x, t)+\gamma r^{2} B(x, t)^{3} \\
& +\alpha B_{x}(x, t) C(x, t)^{2}+A_{x}(x, t) C(x, t)^{2} \\
& +2 \alpha B(x, t) C_{x}(x, t)^{2}+2 \gamma r^{2} A(x, t) B(x, t) C(x, t) \\
& \left.-2 \alpha B_{x}(x, t) C_{x}(x, t) C(x, t)\right)=0,
\end{aligned}
$$

where $A(x, t), B(x, t)$, and $C(x, t)$ are real functions. We separate the imaginary and real parts of (5) and have

$$
\begin{aligned}
& 2 \gamma r^{2} A(x, t) C(x, t)^{2}+2 \alpha A(x, t) C_{x}(x, t)^{2} \\
& \quad+\gamma r^{2} A(x, t)^{3}-2 \alpha A_{x}(x, t) C_{x}(x, t) C(x, t) \\
& \quad+\alpha A_{x x}(x, t) C(x, t)^{2}-\alpha A(x, t) C_{x x}(x, t) C(x, t) \\
& \quad+B(x, t) C(x, t) C_{x}(x, t)+3 \gamma r^{2} A(x, t)^{2} C(x, t) \\
& \quad+\gamma r^{2} B(x, t)^{2} C(x, t)-B_{t}(x, t) C(x, t)^{2} \\
& \quad+\gamma r^{2} A(x, t) B(x, t)^{2}=0, \\
& \gamma r A+(x, t)^{2} B(x, t)-A(x, t) C(x, t) C_{x}(x, t) \\
& \quad-\alpha B(x, t) C_{x x}(x, t) C(x, t)+\gamma r^{2} B(x, t)^{3} \\
& +\alpha B_{x}(x, t) C(x, t)^{2}+A_{x}(x, t) C(x, t)^{2} \\
& +2 \alpha B(x, t) C_{x}(x, t)^{2}+2 \gamma r^{2} A(x, t) B(x, t) C(x, t) \\
& \quad-2 \alpha B_{x}(x, t) C_{x}(x, t) C(x, t)=0 .
\end{aligned}
$$

We suppose that $A(x, t), B(x, t)$, and $C(x, t)$ are the following functions:

$$
\begin{aligned}
A(x, t)= & a_{1} e^{p(V x+K t)}+a_{2} e^{-p(V x+K t)}+a_{3} e^{q(W x+L t)} \\
& +a_{4} e^{-q(W x+L t)}, \\
B(x, t)= & b_{1} e^{p(V x+K t)}+b_{2} e^{-p(V x+K t)}+b_{3} e^{q(W x+L t)} \\
& +b_{4} e^{-q(W x+L t)}, \\
C(x, t)= & c_{1} e^{p(V x+K t)}+c_{2} e^{-p(V x+K t)}+c_{3} e^{q(W x+L t)} \\
& +c_{4} e^{-q(W x+L t)}
\end{aligned}
$$

where $a_{i}, b_{i}, c_{i}(i=1, \ldots, 4), p, q, V, K, W$, and $L$ are the constants to be determined later. We substitute (7) into (6) and equate all coefficients of $e^{m p(V x+K t)} e^{n q(W x+L t)}(m, n=$ $-3, \ldots, 3)$ to 0 , and then we obtain a set of algebraic equations for $a_{i}, b_{i}, c_{i}(i=1, \ldots, 4), p, q, V, K, W$, and $L$. With the aid of Maple, we have

$$
\begin{aligned}
& a_{1}=0, \\
& a_{2}=0, \\
& a_{3}=\frac{b_{4}^{2}}{\sqrt{4 c_{2}^{2}-b_{4}^{2}}}, \\
& a_{4}=\frac{b_{4}^{2}}{\sqrt{4 c_{2}^{2}-b_{4}^{2}}}, \\
& b_{1}=0, \\
& b_{2}=0, \\
& b_{3}=-b_{4}, \\
& c_{1}=c_{2}, \\
& c_{3}=-\frac{2 c_{2}^{2}}{\sqrt{4 c_{2}^{2}-b_{4}^{2}}}, \\
& L=-\frac{2 c_{2}^{2}}{\sqrt{4 c_{2}^{2}-b_{4}^{2}}} \\
& c_{4}=0, \\
& V=\frac{r b_{4} \sqrt{-2(\gamma / \alpha)}}{2 p b_{2} \sqrt{4 c_{2}^{2}-b_{4}^{2}}} \\
& W=0,
\end{aligned}
$$

Substituting (8) and (7) into (4), we can obtain the solution of (3) as follows:

$$
E(x, t)=r e^{i r^{2} \gamma t}\left(1+\frac{b_{4}^{2} \cosh \left(\left(\gamma r^{2} b_{4} \sqrt{4 c_{2}^{2}-b_{4}^{2}} / 2 c_{2}^{2}\right) t\right)+i b_{4} \sqrt{4 c_{2}^{2}-b_{4}^{2}} \sinh \left(\left(\gamma r^{2} b_{4} \sqrt{4 c_{2}^{2}-b_{4}^{2}} / 2 c_{2}^{2}\right) t\right)}{c_{2} \sqrt{4 c_{2}^{2}-b_{4}^{2}} \cosh \left(\left(r \sqrt{-2(\gamma / \alpha)} b_{4} / 2 c_{2}\right) x\right)-2 c_{2}^{2} \cosh \left(\left(\gamma r^{2} b_{4} \sqrt{4 c_{2}^{2}-b_{4}^{2}} / 2 c_{2}^{2}\right) t\right)}\right) .
$$


Finally, according to $n=\left(\beta k_{1}^{2} /\left(k_{2}^{2}-c_{s}^{2} k_{1}^{2}\right)\right)|E|^{2}$, we obtain the exact solution of (1) as follows:

$$
\begin{aligned}
& E(x, t)=r e^{i r^{2} \gamma t}\left(1+\frac{b_{4}^{2} \cosh \left(\left(\gamma r^{2} b_{4} \sqrt{4 c_{2}^{2}-b_{4}^{2}} / 2 c_{2}^{2}\right) t\right)+i b_{4} \sqrt{4 c_{2}^{2}-b_{4}^{2}} \sinh \left(\left(\gamma r^{2} b_{4} \sqrt{4 c_{2}^{2}-b_{4}^{2}} / 2 c_{2}^{2}\right) t\right)}{c_{2} \sqrt{4 c_{2}^{2}-b_{4}^{2}} \cosh \left(\left(r \sqrt{-2(\gamma / \alpha)} b_{4} / 2 c_{2}\right) x\right)-2 c_{2}^{2} \cosh \left(\left(\gamma r^{2} b_{4} \sqrt{4 c_{2}^{2}-b_{4}^{2}} / 2 c_{2}^{2}\right) t\right)}\right), \\
& n(x, t)=\frac{\beta k_{1}^{2}}{k_{2}^{2}-c_{s}^{2} k_{1}^{2}}|E(x, t)|^{2},
\end{aligned}
$$

where $\gamma=-\delta \beta k_{1}^{2} /\left(k_{2}^{2}-c_{s}^{2} k_{1}^{2}\right)$. Here (10) is a unified solution formula of (1) which can produce periodic wave solution, bisoliton solution, and a series of breather solutions.

\section{Exact Periodic Wave, Bisoliton, and Various} Breather Solutions for (1)

In this section, according to (10), when we select different parameters in (10), we can obtain different structure exact solutions of (1) as follows.
Case 1 (the periodic wave solution). When $\alpha \gamma<0$, we set $b_{4}=i b$, and we obtain the following periodic solution:

$$
\begin{aligned}
& E(x, t)=r e^{i r^{2} \gamma t}\left(1-\frac{b^{2} \cos \left(\left(\gamma r^{2} b \sqrt{4 c_{2}^{2}+b^{2}} / 2 c_{2}^{2}\right) t\right)+i b \sqrt{4 c_{2}^{2}+b^{2}} \sin \left(\left(\gamma r^{2} b \sqrt{4 c_{2}^{2}+b^{2}} / 2 c_{2}^{2}\right) t\right)}{c_{2} \sqrt{4 c_{2}^{2}+b^{2}} \cos \left(\left(r \sqrt{-2(\gamma / \alpha)} b / 2 c_{2}\right) x\right)-2 c_{2}^{2} \cos \left(\left(\gamma r^{2} b \sqrt{4 c_{2}^{2}+b^{2}} / 2 c_{2}^{2}\right) t\right)}\right), \\
& n(x, t)=\frac{\beta k_{1}^{2}}{k_{2}^{2}-c_{s}^{2} k_{1}^{2}}|E(x, t)|^{2} .
\end{aligned}
$$

Case 2 (the bisoliton solution). When $\alpha \gamma<0$ and $4 c_{2}^{2}-b_{4}^{2}>0$,

we obtain the following bisoliton solution:

$$
\begin{aligned}
& E(x, t)=r e^{i r^{2} \gamma t}\left(1+\frac{b_{4}^{2} \cosh \left(\left(\gamma r^{2} b_{4} \sqrt{4 c_{2}^{2}-b_{4}^{2}} / 2 c_{2}^{2}\right) t\right)+i b_{4} \sqrt{4 c_{2}^{2}-b_{4}^{2}} \sinh \left(\left(\gamma r^{2} b_{4} \sqrt{4 c_{2}^{2}-b_{4}^{2}} / 2 c_{2}^{2}\right) t\right)}{c_{2} \sqrt{4 c_{2}^{2}-b_{4}^{2}} \cosh \left(\left(r \sqrt{-2(\gamma / \alpha)} b_{4} / 2 c_{2}\right) x\right)-2 c_{2}^{2} \cosh \left(\left(\gamma r^{2} b_{4} \sqrt{4 c_{2}^{2}-b_{4}^{2}} / 2 c_{2}^{2}\right) t\right)}\right), \\
& n(x, t)=\frac{\beta k_{1}^{2}}{k_{2}^{2}-c_{s}^{2} k_{1}^{2}}|E(x, t)|^{2} .
\end{aligned}
$$

Case 3 (various breather solutions). (1) When $\alpha \gamma>0$ and $4 c_{2}^{2}-b_{4}^{2}>0$, we obtain the following Akhmediev breather solution:

$$
\begin{aligned}
& E(x, t)=r e^{i r^{2} \gamma t}\left(1+\frac{b_{4}^{2} \cosh \left(\left(\gamma r^{2} b_{4} \sqrt{4 c_{2}^{2}-b_{4}^{2}} / 2 c_{2}^{2}\right) t\right)+i b_{4} \sqrt{4 c_{2}^{2}-b_{4}^{2}} \sinh \left(\left(\gamma r^{2} b_{4} \sqrt{4 c_{2}^{2}-b_{4}^{2}} / 2 c_{2}^{2}\right) t\right)}{c_{2} \sqrt{4 c_{2}^{2}-b_{4}^{2}} \cos \left(\left(r \sqrt{-2(\gamma / \alpha)} b_{4} / 2 c_{2}\right) x\right)-2 c_{2}^{2} \cosh \left(\left(\gamma r^{2} b_{4} \sqrt{4 c_{2}^{2}-b_{4}^{2}} / 2 c_{2}^{2}\right) t\right)}\right), \\
& n(x, t)=\frac{\beta k_{1}^{2}}{k_{2}^{2}-c_{s}^{2} k_{1}^{2}}|E(x, t)|^{2} .
\end{aligned}
$$




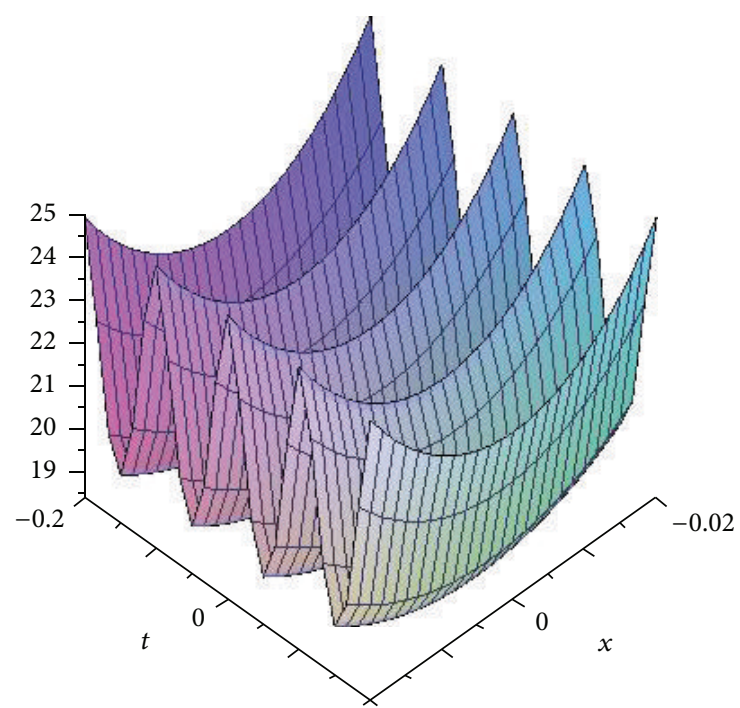

FIgURE 1: The 3D graphics of $|E(x, t)|$ in (11) $(\alpha=-2, \beta=1, \delta=-1$, $c_{s}=1, \gamma=4, k_{1}=2, k_{2}=\sqrt{5}, r=2, b=2$, and $\left.c_{2}=0.2\right)$.

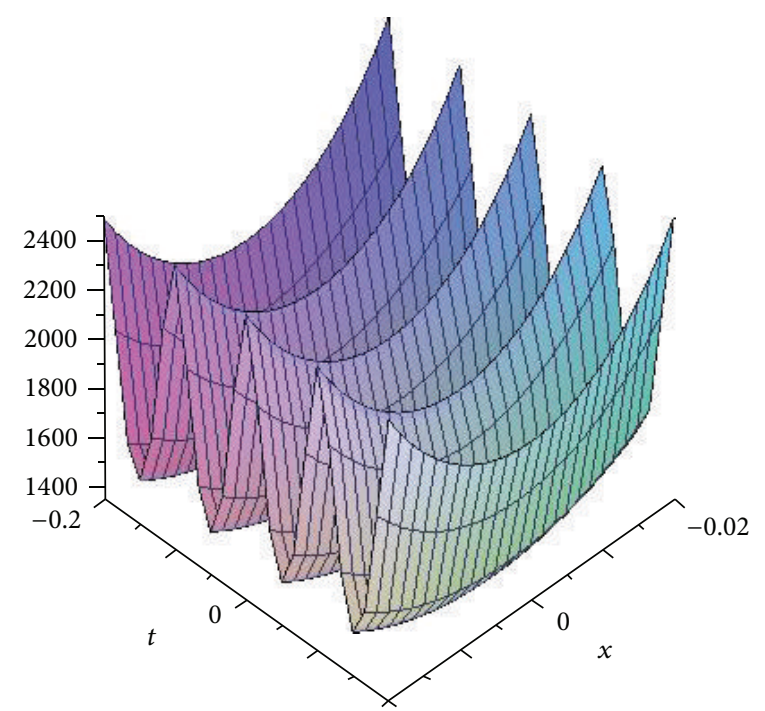

FIgURE 2: The 3D graphics of $n(x, t)$ in (11) $(\alpha=-2, \beta=1, \delta=-1$, $c_{s}=1, \gamma=4, k_{1}=2, k_{2}=\sqrt{5}, r=2, b=2$, and $\left.c_{2}=0.2\right)$.

(2) When $\alpha \gamma>0$, we set $b_{4}=i b$, and we obtain the following Ma breather solution:

$$
\begin{aligned}
& E(x, t)=r e^{i r^{2} \gamma t}\left(1-\frac{b^{2} \cos \left(\left(\gamma r^{2} b \sqrt{4 c_{2}^{2}+b^{2}} / 2 c_{2}^{2}\right) t\right)+i b \sqrt{4 c_{2}^{2}+b^{2}} \sin \left(\left(\gamma r^{2} b \sqrt{4 c_{2}^{2}+b^{2}} / 2 c_{2}^{2}\right) t\right)}{c_{2} \sqrt{4 c_{2}^{2}+b^{2}} \cosh \left(\left(r \sqrt{-2(\gamma / \alpha)} b / 2 c_{2}\right) x\right)-2 c_{2}^{2} \cos \left(\left(\gamma r^{2} b \sqrt{4 c_{2}^{2}+b^{2}} / 2 c_{2}^{2}\right) t\right)}\right), \\
& n(x, t)=\frac{\beta k_{1}^{2}}{k_{2}^{2}-c_{s}^{2} k_{1}^{2}}|E(x, t)|^{2} .
\end{aligned}
$$

(3) When $\alpha \gamma>0$, we set $c_{2}>0$ and $b_{4} \rightarrow 0$, and we obtain the following Peregrine breather solution:

$$
\begin{aligned}
& E(x, t)=r e^{i r^{2} \gamma t}\left(1-\frac{\alpha+i 2 \alpha \gamma r^{2} t}{r^{2} \gamma x^{2}+\alpha \gamma^{2} r^{4} t^{2}}\right), \\
& n(x, t)=\frac{\beta k_{1}^{2}}{k_{2}^{2}-c_{s}^{2} k_{1}^{2}}|E(x, t)|^{2} .
\end{aligned}
$$

To verify the correctness of our results, by using the numerical simulation method, the 3D graphics of the periodic waves, bisolitons, and various breathers of (1) are given in Figures 1-10.

\section{Conclusion}

To summarize, by using the Exp-function method, we have obtained the periodic wave solution, bisoliton solution, and various breather solutions of the Zakharov equations. Firstly, by using the appropriate transformation, the Zakharov equations are transformed into the $(1+1)$-dimensional nonlinear
Schrödinger equation. Then, based on the Exp-function method, a unified solution formula of the $(1+1)$-dimensional nonlinear Schrödinger equation is given. Under different parameter conditions, the exact solutions of the Zakharov equations which contain the periodic wave solution, bisoliton solution, Akhmediev breather solution, Ma breather solution, and Peregrine breather solution are obtained. The results greatly enrich the diversity of exact solutions of the Zakharov equations.

Secondly, based on the numerical simulations of these solutions, we obtain some physics behind our results. Figures 3 and 4 show the bisolitons which move to each other; they meet and have an elastic collision. When the elastic collision occurs, the amplitude is obviously increased. After separation, they do the reverse motion. Figures 5 and 6 show Akhmediev breather; it yields the breather which affects spatial direction. Figures 7 and 8 show Ma breather; it yields the breather which affects time direction. Figures 9 and 10 show Peregrine breather which is a high amplitude wave. With the increase of time, the wavelength becomes larger, and the amplitude decreases gradually. Finally, it becomes a plane wave. 


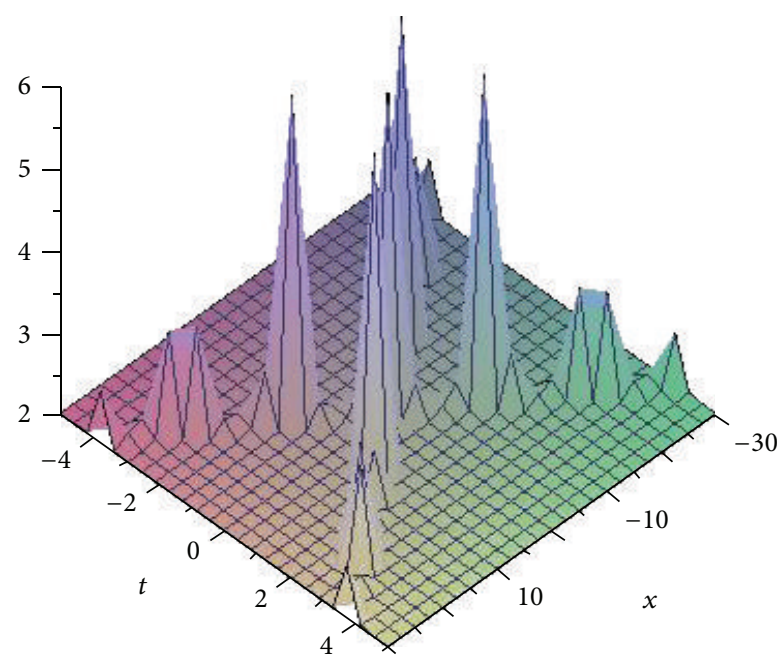

Figure 3: The 3D graphics of $|E(x, t)|$ in (12) $(\alpha=-2, \beta=1, \delta=-1$, $c_{s}=1, \gamma=4, k_{1}=2, k_{2}=\sqrt{5}, r=2, b_{4}=2$, and $\left.c_{2}=5\right)$.

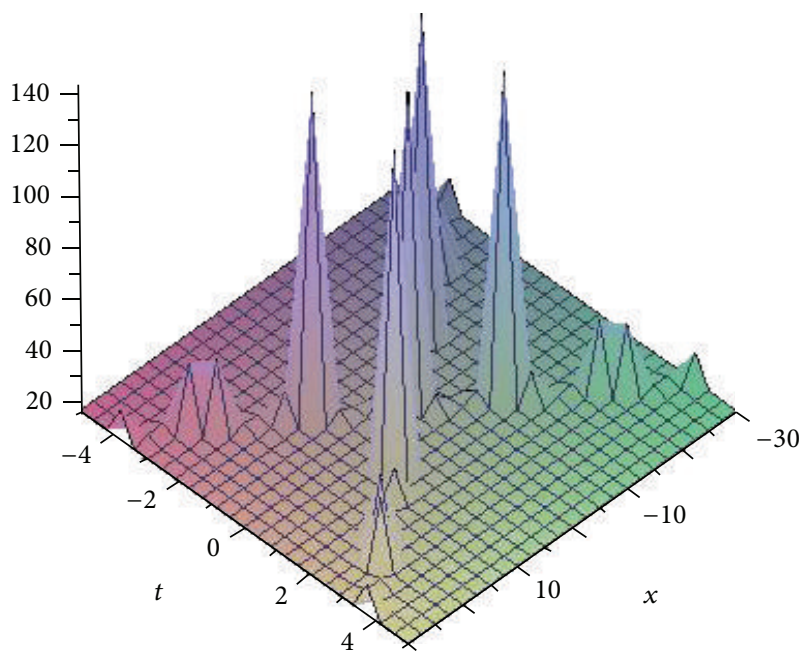

FIGURE 4: The 3D graphics of $n(x, t)$ in (12) $(\alpha=-2, \beta=1, \delta=-1$, $c_{s}=1, \gamma=4, k_{1}=2, k_{2}=\sqrt{5}, r=2, b_{4}=2$, and $\left.c_{2}=5\right)$.

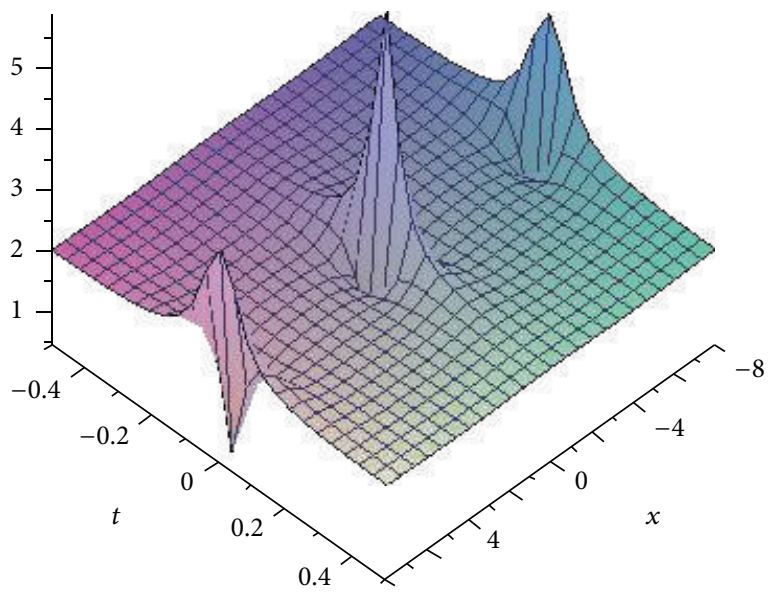

Figure 5: The 3D graphics of $|E(x, t)|$ in (13) $(\alpha=-2, \beta=1, \delta=1$, $c_{s}=1, \gamma=-4, k_{1}=2, k_{2}=\sqrt{5}, r=2, b_{4}=2$, and $\left.c_{2}=5\right)$.

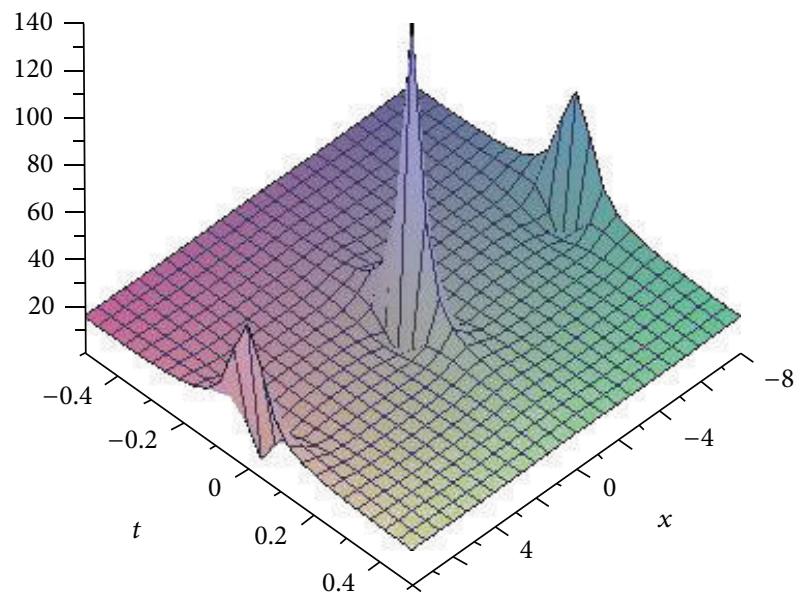

Figure 6: The 3D graphics of $n(x, t)$ in (13) $(\alpha=-2, \beta=1, \delta=1$, $c_{s}=1, \gamma=-4, k_{1}=2, k_{2}=\sqrt{5}, r=2, b_{4}=2$, and $\left.c_{2}=5\right)$.

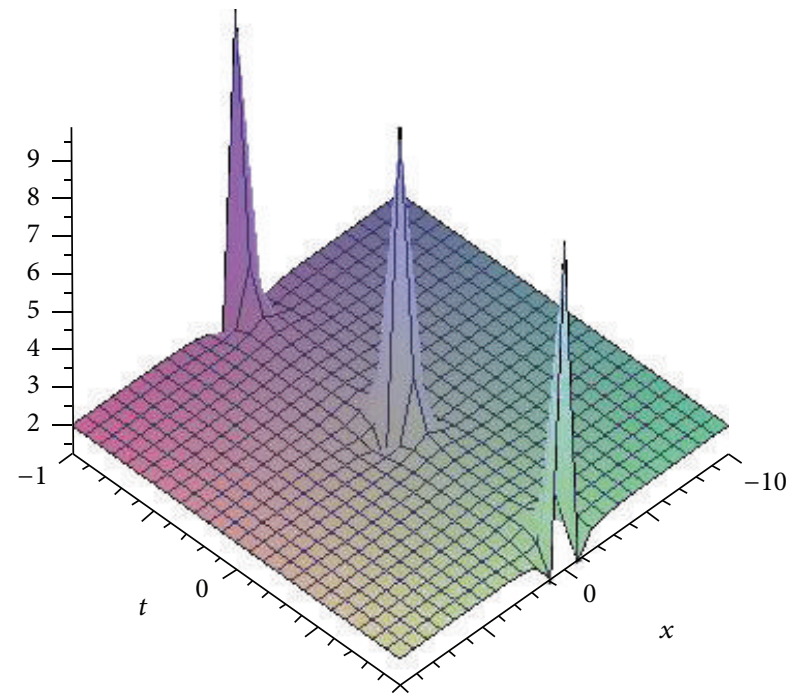

Figure 7: The 3D graphics of $|E(x, t)|$ in (14) $(\alpha=-2, \beta=1, \delta=1$, $c_{s}=1, \gamma=-4, k_{1}=2, k_{2}=\sqrt{5}, r=2, b=2$, and $\left.c_{2}=5\right)$.

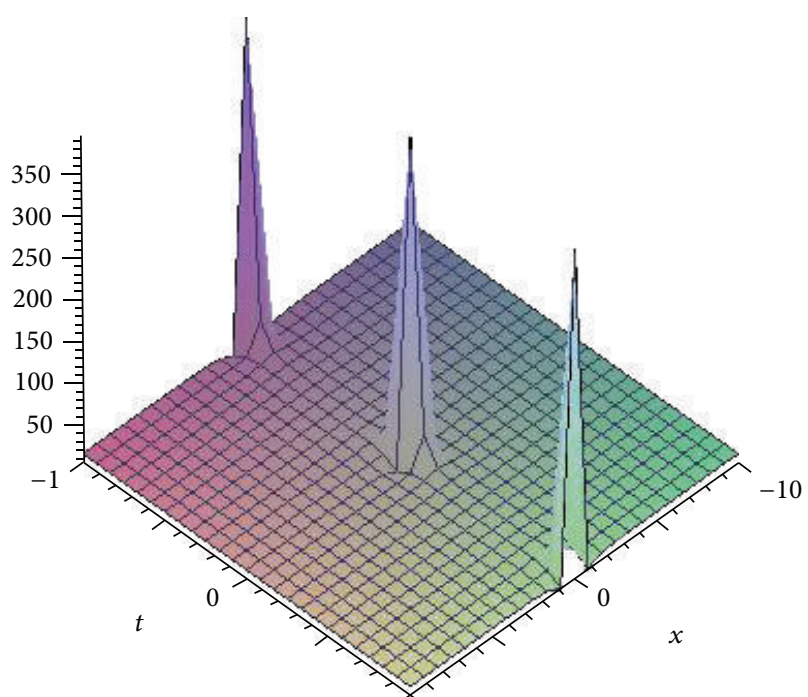

FIgURE 8: The 3D graphics of $n(x, t)$ in (14) $(\alpha=-2, \beta=1, \delta=1$, $c_{s}=1, \gamma=-4, k_{1}=2, k_{2}=\sqrt{5}, r=2, b=2$, and $\left.c_{2}=5\right)$. 


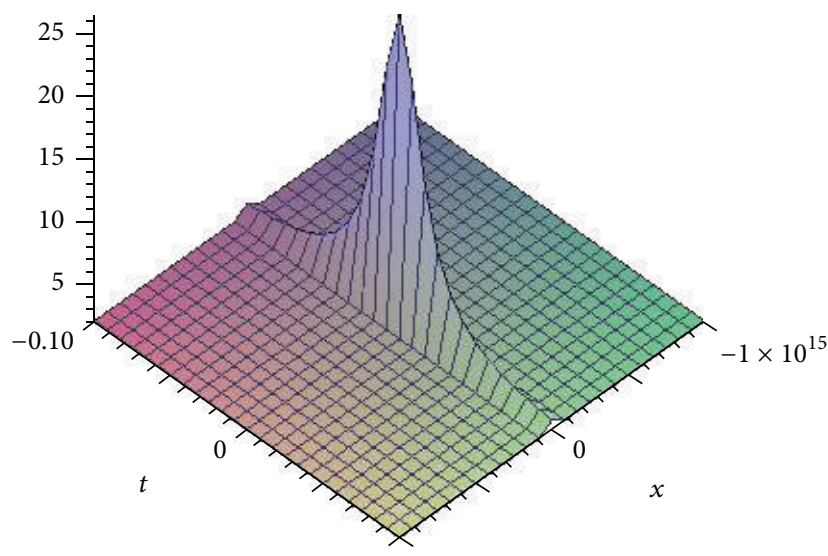

Figure 9: The 3D graphics of $|E(x, t)|$ in (15) $(\alpha=-2, \beta=1, \delta=1$, $c_{s}=1, \gamma=-4, k_{1}=2, k_{2}=\sqrt{5}, r=2$, and $\left.c_{2}=5\right)$.

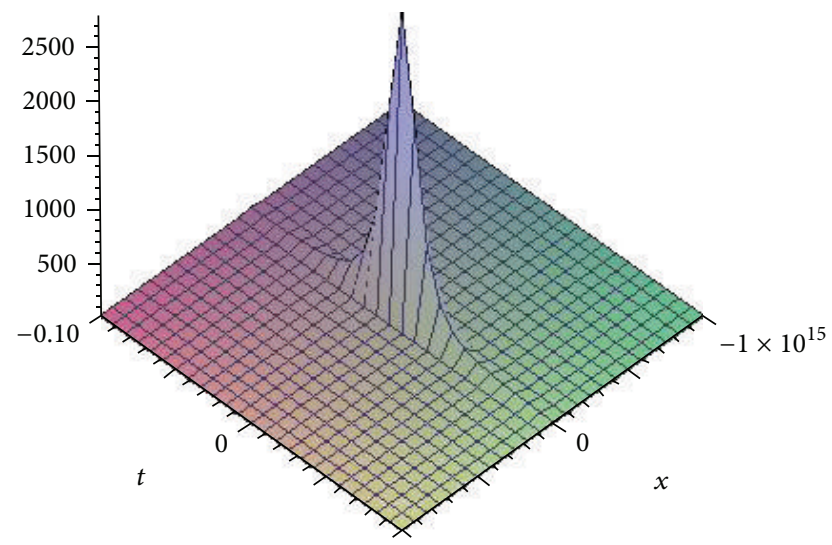

Figure 10: The 3D graphics of $n(x, t)$ in (15) $(\alpha=-2, \beta=1, \delta=1$, $c_{s}=1, \gamma=-4, k_{1}=2, k_{2}=\sqrt{5}, r=2$, and $\left.c_{2}=5\right)$.

Finally, when $\alpha=1 / 2$ and $\gamma=1$, based on the work of Akhmediev et al. $[19,20]$, we can obtain the following higherorder rational rogue wave solutions of (3):

$$
E(x, t)=\sqrt{\frac{1}{\gamma}}\left(1-\frac{G+i H}{D}\right) e^{i t}, \quad \gamma>0,
$$

where $G=\left(4 x^{2}+12 \alpha x^{2}+72 \alpha^{2} t^{2}+48 \alpha x^{2} t^{2}+80 \alpha^{2} t^{4}-\right.$ $\left.3 \alpha^{2}\right) / 16 \alpha^{2}, H=t\left(4 x^{2}+8 \alpha t^{2}+16 \alpha x^{2} t^{2}+16 \alpha^{2} t^{4}-15 \alpha^{2}-\right.$ $\left.12 \alpha x^{2}\right) / 8 \alpha^{2}$, and $D=\left(8 x^{6}+\alpha\left(12+48 t^{2}\right) x^{4}+6 \alpha^{2}\left(4 t^{2}-3\right)^{2} x^{2}+\right.$ $\left.\alpha^{3}\left(9+64 t^{6}+432 t^{4}+396 t^{2}\right)\right) / 192 \alpha^{3}$. Then we have the following higher-order rational rogue wave solutions of (1):

$$
\begin{aligned}
& E(x, t)=\sqrt{\frac{1}{\gamma}}\left(1-\frac{G+i H}{D}\right) e^{i t}, \\
& n(x, t)=\frac{\beta k_{1}^{2}}{k_{2}^{2}-c_{s}^{2} k_{1}^{2}}|E(x, t)|^{2} .
\end{aligned}
$$

\section{Conflict of Interests}

The authors declare that there is no conflict of interests regarding the publication of this paper.

\section{References}

[1] Y. Shang and X. Zheng, "The first-integral method and abundant explicit exact solutions to the Zakharov equations," Journal of Applied Mathematics, vol. 2012, Article ID 818345, 16 pages, 2012.

[2] Y. Shang, Y. Huang, and W. Yuan, “The extended hyperbolic functions method and new exact solutions to the Zakharov equations," Applied Mathematics and Computation, vol. 200, no. 1, pp. 110-122, 2008.

[3] D. J. Huang and H. Q. Zhang, "Extended hyperbolic function method and new exact solitary wave solutions of Zakharov equations," Acta Physica Sinica, vol. 53, no. 8, pp. 2434-2438, 2004.

[4] S. D. Liu, Z. T. Fu, S. K. Liu, and Q. Zhao, "Enveloping periodic solutions to nonlinear wave equations with Jacobi elliptic functions," Acta Physica Sinica, vol. 51, no. 4, pp. 718-722, 2002.

[5] G. Wu, M. Zhang, L. Shi, W. Zhang, and J. Han, "An extended expansion method or Jacobi elliptic functions and new exact periodic solutions of Zakharov equations," Acta Physica Sinica, vol. 56, no. 9, pp. 5054-5059, 2007.

[6] C. H. Zhao and Z. M. Sheng, "Explicit travelling wave solutions for Zakharov equations," Acta Physica Sinica, vol. 53, no. 6, pp. 1629-1634, 2004.

[7] H. L. Zhen, B. Tian, Y. F. Wang et al., "Soliton solutions and chaotic motions of the Zakharov equations for the Langmuir wave in the plasma," Physics of Plasmas, vol. 22, no. 3, Article ID 032307, 2015.

[8] X. Y. Gao, "Variety of the cosmic plasmas: general variablecoefficient Korteweg-de Vries-Burgers equation with experimental/observational support," EPL, vol. 110, no. 1, Article ID 15002, p. 15002, 2015.

[9] X. Y. Gao, "Comment on 'Solitons, Bäcklund transformation, and Lax pair for the $(2+1)$-dimensional Boiti-Leon- Pempinelli equation for the water waves' [J. Math. Phys. 51, 093519 (2010)]," Journal of Mathematical Physics, vol. 56, no. 1, Article ID 014101, 2015.

[10] X.-Y. Gao, "Bäcklund transformation and shock-wave-type solutions for a generalized $(3+1)$-dimensional variable-coefficient B-type Kadomtsev-Petviashvili equation in fluid mechanics," Ocean Engineering, vol. 96, pp. 245-247, 2015.

[11] X. Y. Gao, "Incompressible-fluid symbolic computation and Bäcklund transformation: $(3+1)$-dimensional variable-coefficient Boiti-Leon-Manna-Pempinelli model," Zeitschrift für Naturforschung A, vol. 70, pp. 59-61, 2015.

[12] Y.-F. Wang, B. Tian, M. Wang, and H.-L. Zhen, "Solitons via an auxiliary function for an inhomogeneous higher-order nonlinear Schrödinger equation in optical fiber communications," Nonlinear Dynamics, vol. 79, no. 1, pp. 721-729, 2015.

[13] W.-R. Sun, B. Tian, Y. Jiang, and H.-L. Zhen, "Optical rogue waves associated with the negative coherent coupling in an isotropic medium," Physical Review E, vol. 91, no. 2, Article ID 023205, 2015.

[14] H.-F. Zhang, H.-Q. Hao, and J.-W. Zhang, "Breathers and soliton solutions for a generalization of the nonlinear Schrödinger equation," Mathematical Problems in Engineering, vol. 2013, Article ID 456864, 5 pages, 2013.

[15] C. Wang, Z. Dai, and C. Liu, "The breather-like and rational solutions for the integrable kadomtsev-petviashvili-based system," Advances in Mathematical Physics, vol. 2015, Article ID 861069, 7 pages, 2015. 
[16] F. Fedele, "Rogue waves in oceanic turbulence," Physica D: Nonlinear Phenomena, vol. 237, no. 14-17, pp. 2127-2131, 2008.

[17] A. Zaviyalov, O. Egorov, R. Iliew, and F. Lederer, "Rogue waves in mode-locked fiber lasers," Physical Review A-Atomic, Molecular, and Optical Physics, vol. 85, Article ID 013828, 6 pages, 2012.

[18] C. Bonatto, M. Feyereisen, S. Barland et al., "Deterministic optical rogue waves," Physical Review Letters, vol. 107, no. 5, Article ID 053901, 5 pages, 2011.

[19] N. Akhmediev, A. Ankiewicz, and M. Taki, "Waves that appear from nowhere and disappear without a trace," Physics Letters A, vol. 373, no. 6, pp. 675-678, 2009.

[20] N. Akhmediev, J. M. Soto-Crespo, and A. Ankiewicz, "Extreme waves that appear from nowhere: on the nature of rogue waves," Physics Letters A, vol. 373, no. 25, pp. 2137-2145, 2009. 


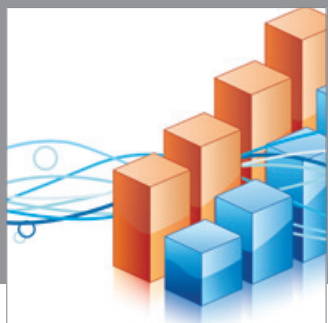

Advances in

Operations Research

mansans

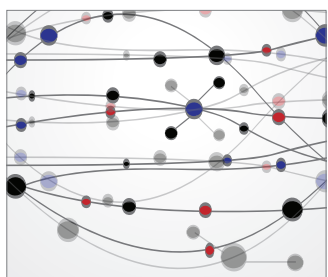

The Scientific World Journal
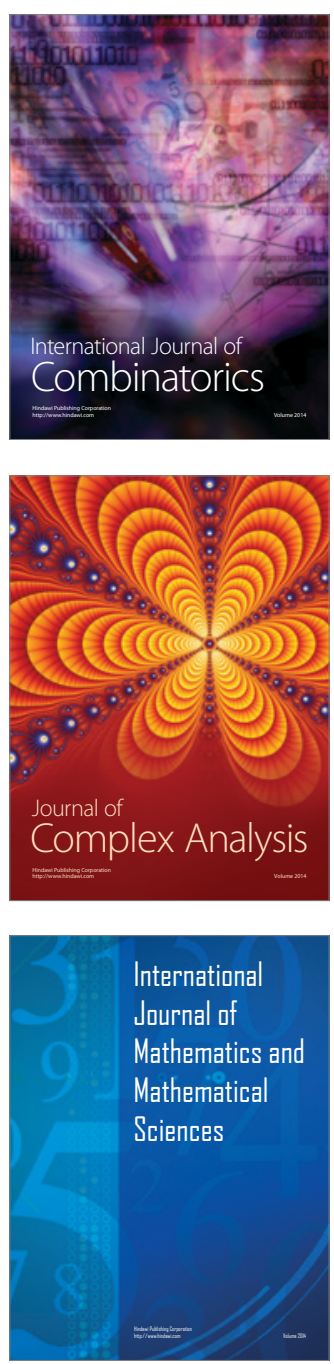
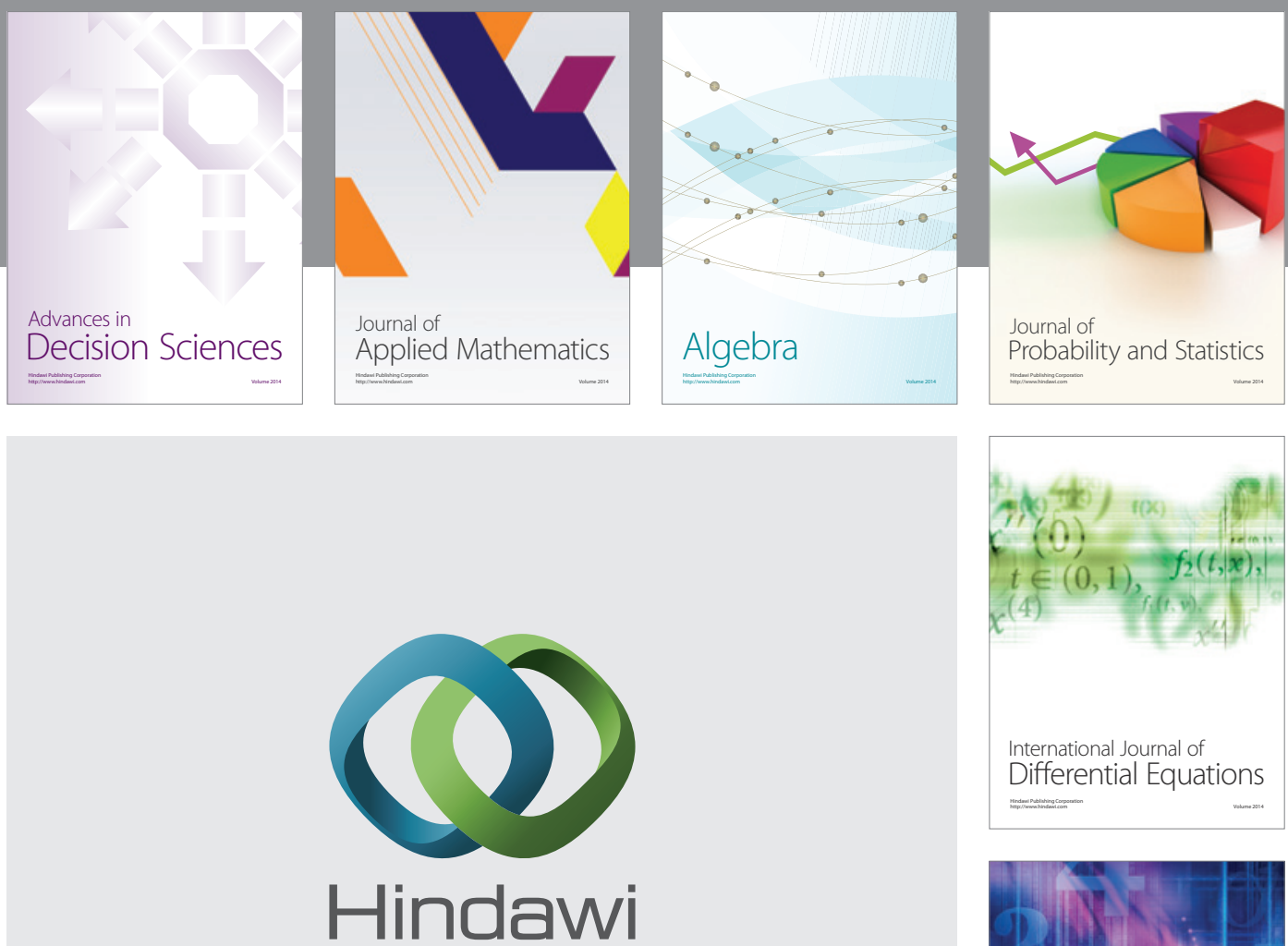

Submit your manuscripts at http://www.hindawi.com
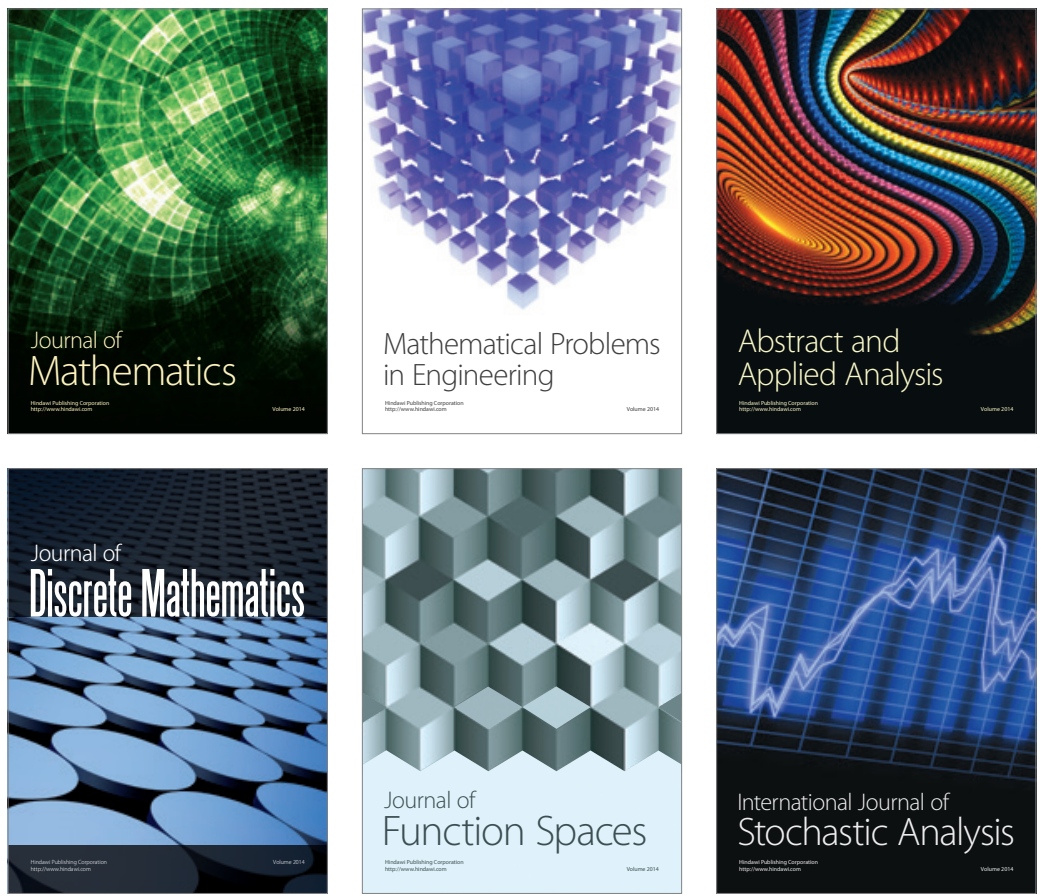

Journal of

Function Spaces

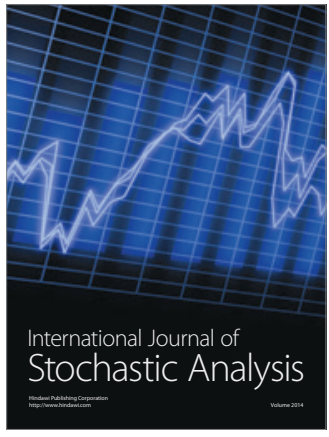

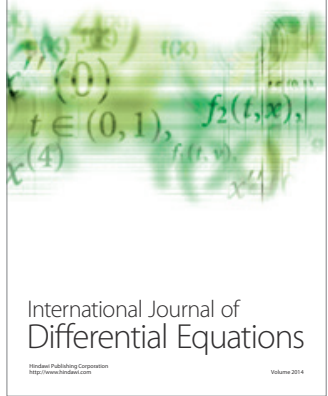
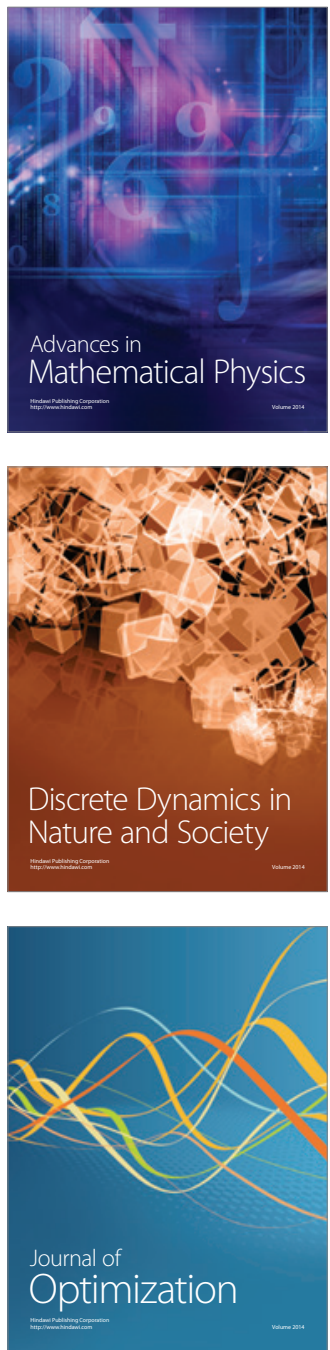